\title{
Pedicle screw placement accuracy using ultra-low radiation imaging with image enhancement versus conventional fluoroscopy in minimally invasive transforaminal lumbar interbody fusion: an internally randomized controlled trial
}

\author{
Gautam Nayar, BS, ${ }^{1}$ Daniel J. Blizzard, MD, ${ }^{2}$ Timothy Y. Wang, MD, ${ }^{1}$ Steven Cook, MD, ${ }^{1}$ \\ Adam G. Back, MD, ${ }^{1}$ David Vincent, MD, ${ }^{3}$ and Isaac O. Karikari, MD ${ }^{1}$
}

Departments of ${ }^{1}$ Neurological Surgery and ${ }^{2}$ Orthopedic Surgery, Duke University Medical Center, Durham, North Carolina; and ${ }^{3}$ Neurosurgical Specialists, Norfolk, Virginia

OBJECTIVE A previous study found that ultra-low radiation imaging (ULRI) with image enhancement significantly decreases radiation exposure by roughly $75 \%$ for both the patient and operating room personnel during minimally invasive transforaminal lumbar interbody fusion (MIS-TLIF) $(p<0.001)$. However, no clinical data exist on whether this imaging modality negatively impacts patient outcomes. Thus, the goal of this randomized controlled trial was to assess pedicle screw placement accuracy with ULRI with image enhancement compared with conventional, standard-dose fluoroscopy for patients undergoing single-level MIS-TLIF.

METHODS An institutional review board-approved, prospective internally randomized controlled trial was performed to compare breach rates for pedicle screw placement performed using ULRI with image enhancement versus conventional fluoroscopy. For cannulation and pedicle screw placement, surgery on 1 side (left vs right) was randomly assigned to be performed under ULRI. Screws on the opposite side were placed under conventional fluoroscopy, thereby allowing each patient to serve as his/her own control. In addition to standard intraoperative images to check screw placement, each patient underwent postoperative CT. Three experienced neurosurgeons independently analyzed the images and were blinded as to which imaging modality was used to assist with each screw placement. Screw placement was analyzed for pedicle breach (lateral vs medial and Grade 0 [<2.0 mm], Grade 1 [2.0-4.0 mm], or Grade 2 [> $4.0 \mathrm{~mm}]$ ), appropriate screw depth (50\%-75\% of the vertebral body's anteroposterior dimension), and appropriate screw angle (within $10^{\circ}$ of the pedicle angle). The effective breach rate was calculated as the percentage of screws evaluated as breached $>2.0$ $\mathrm{mm}$ medially or postoperatively symptomatic.

RESULTS Twenty-three consecutive patients underwent single-level MIS-TLIF, and their sides were randomly assigned to receive ULRI. No patient had immediate postoperative complications (e.g., neurological decline, need for hardware repositioning). On CT confirmation, 4 screws that had K-wire placement and cannulation under ULRI and screw placement under conventional fluoroscopy showed deviations. There were 2 breaches that deviated medially but both were Grade $0(<2.0 \mathrm{~mm})$. Similarly, 2 breaches occurred that were Grade $1(>2.0 \mathrm{~mm})$ but both deviated laterally. Therefore, the effective breach rate (breach $>2.0 \mathrm{~mm}$ deviated medially) was unchanged in both imaging groups ( $0 \%$ using either ULRI or conventional fluoroscopy; $p=1.00$ ).

CONCLUSIONS ULRI with image enhancement does not compromise accuracy during pedicle screw placement compared with conventional fluoroscopy while it significantly decreases radiation exposure to both the patient and operating room personnel.

https://thejns.org/doi/abs/10.3171/2017.5.SPINE17123

KEY WORDS ultra-low radiation imaging; pedicle screw accuracy; minimally invasive; transforaminal lumbar interbody fusion

ABBREVIATIONS BMI = body mass index; MIS-TLIF = minimally invasive transforaminal lumbar interbody fusion; ULRI = ultra-low radiation imaging . SUBMITTED January 26, 2017. ACCEPTED May 30, 2017.

INCLUDE WHEN CITING Published online December 1, 2017; DOI: 10.3171/2017.5.SPINE17123. 
$\mathrm{M}$ INIMALLY invasive transforaminal lumbar interbody fusion (MIS-TLIF) is an increasingly prevalent and effective treatment for adult spine deformity but with compromised direct visualization of the surgical window. In this setting, intraoperative imaging aids safe hardware placement, thereby leading to significantly decreased rates of pedicle screw breach. ${ }^{8}$ Various imaging modalities, such as 2D fluoroscopy, 3D fluoroscopy, and perioperative CT, have been shown to be effective at reducing the incidence of improperly placed pedicle screws and interbody grafts. ${ }^{5,16}$ Compared with open-approach spine surgery, there is an increased usage of intraoperative imaging in minimally invasive procedures. This results in increased radiation exposure, which has been shown to increase the rates of malignancy and other radiation-related morbidities in patients and health care providers. ${ }^{1,3,6,12,19}$

We have previously shown that ultra-low radiation imaging (ULRI) significantly decreases intraoperative radiation exposure (Table 1), but a decrease in radiation results in sacrifices to image quality (Fig. 1A), often to the point where it may be unsafe to guide hardware placement. ${ }^{21}$ Image enhancement software, such as LessRay (NuVasive, Inc.), can significantly improve image quality (Fig. 1B), but to date there are no clinical data on the impact of software on correct pedicle screw placement.

Therefore, we performed a randomized, prospective, internally controlled study to compare pedicle breach rates in single-level MIS-TLIF performed using ULRI with image enhancement or traditional C-arm fluoroscopy.

\section{Methods \\ Study Design}

This study is an institutional review board-approved, prospective, internally randomized controlled trial of patients undergoing single-level MIS-TLIF performed by a single surgeon. The outcome of interest was the accuracy of percutaneous pedicle screw placement under standarddose fluoroscopy versus ULRI with image enhancement. The inclusion criteria required that patients 1) were $>18$ years, 2) had single-level lumbar disease, 3) underwent single-level MIS-TLIF with percutaneous pedicle screw placement, and 4) had interpretable imaging. Preoperatively collected data included patient age, patient sex, side of TLIF, weight, body mass index (BMI), and spinal levels. All patients underwent presurgical baseline imaging performed using standard C-arm fluoroscopy.

\section{Surgical Technique}

All operations were performed by a single surgeon in
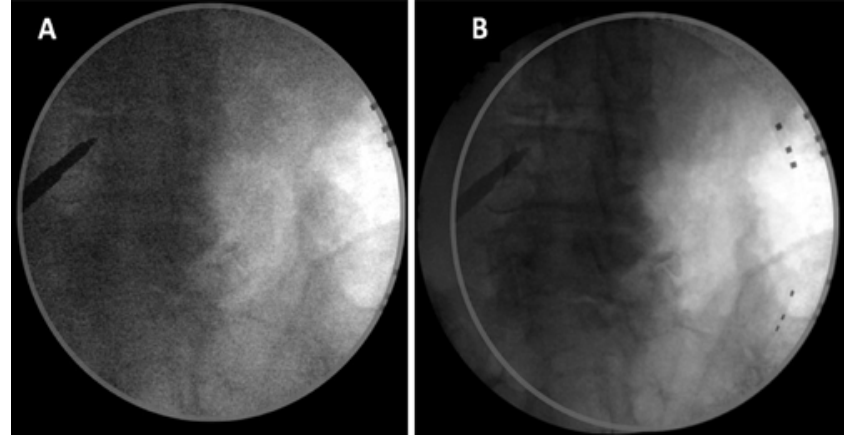

FIG. 1. Ultra-low radiation fluoroscopic images taken during surgery before $(\mathbf{A})$ and after $(B)$ image processing with enhancement. The initial image is grainy, unclear, and compromises the view of relevant surgical anatomy and tools in the operative field, such as the Jamshidi needle (BD Worldwide) impacted into the pedicle of the L-4 vertebral body. Image enhancement (B) improves visualization of the bony anatomy and helps ensure safe instrumentation.

the same operating room. MIS-TLIF was performed using the NuVasive Maximal Access Surgery system, which provides a minimally invasive pedicle-based approach that utilizes a split-blade retractor to access the transforaminal space. After establishing the surgical window, the surgeon then performed the standard components of MISTLIF including annulotomy, discectomy, and intervertebral grafting. NuVasive Precept percutaneous screws were placed on the exposed pedicle under imaging guidance. Contralateral screws were then similarly placed after exposing the pedicle using a small $3.5-\mathrm{cm}$ incision from the midline.

\section{Screw Placement With Image Guidance}

Randomization was accomplished by first dividing screw placement into 3 intervals: 1) left-sided pedicle cannulation and K-wire placement, 2) right-sided pedicle cannulation and K-wire placement, and 3) bilateral pedicle screw placement. Patients were randomly assigned to 2 equal groups to alternate between standard-dose fluoroscopy and ULRI. Patients in the "heads" group underwent ULRI during Intervals 1 and 3 and standard fluoroscopy during Interval 2. Patients in the "tails" group underwent standard fluoroscopy during Intervals 1 and 3 and ULRI during Interval 2 . This resulted in each imaging modality being used to place screws on 1 side, which ensured that the patients would serve as their own controls (Fig. 2). Such internal randomization is important for controlling for body habitus, which can impact imaging utilization and anatomy visualization. Patients were divided into the heads or tails groups by randomly selecting from a con-

TABLE 1. Radiation exposure in patients undergoing single-level MIS-TLIF with intraoperative imaging

\begin{tabular}{lcrrr}
\hline \multicolumn{1}{c}{ Procedure } & Standard-Dose Fluoroscopy $(\mathrm{mGy})^{*}$ & ULRI w/ Image Enhancement (mGy) & Change $(\%)$ & $p$ Value \\
\hline K-wire placement \& cannulation & 34.84 & 7.59 & -78.22 & $<0.001 \dagger$ \\
\hline Pedicle screw placement & 33.90 & 17.39 & -87.43 & $<0.001 \dagger$ \\
\hline
\end{tabular}

* Mean values are shown.

$\dagger$ Statistically significant at $p<0.05$. 

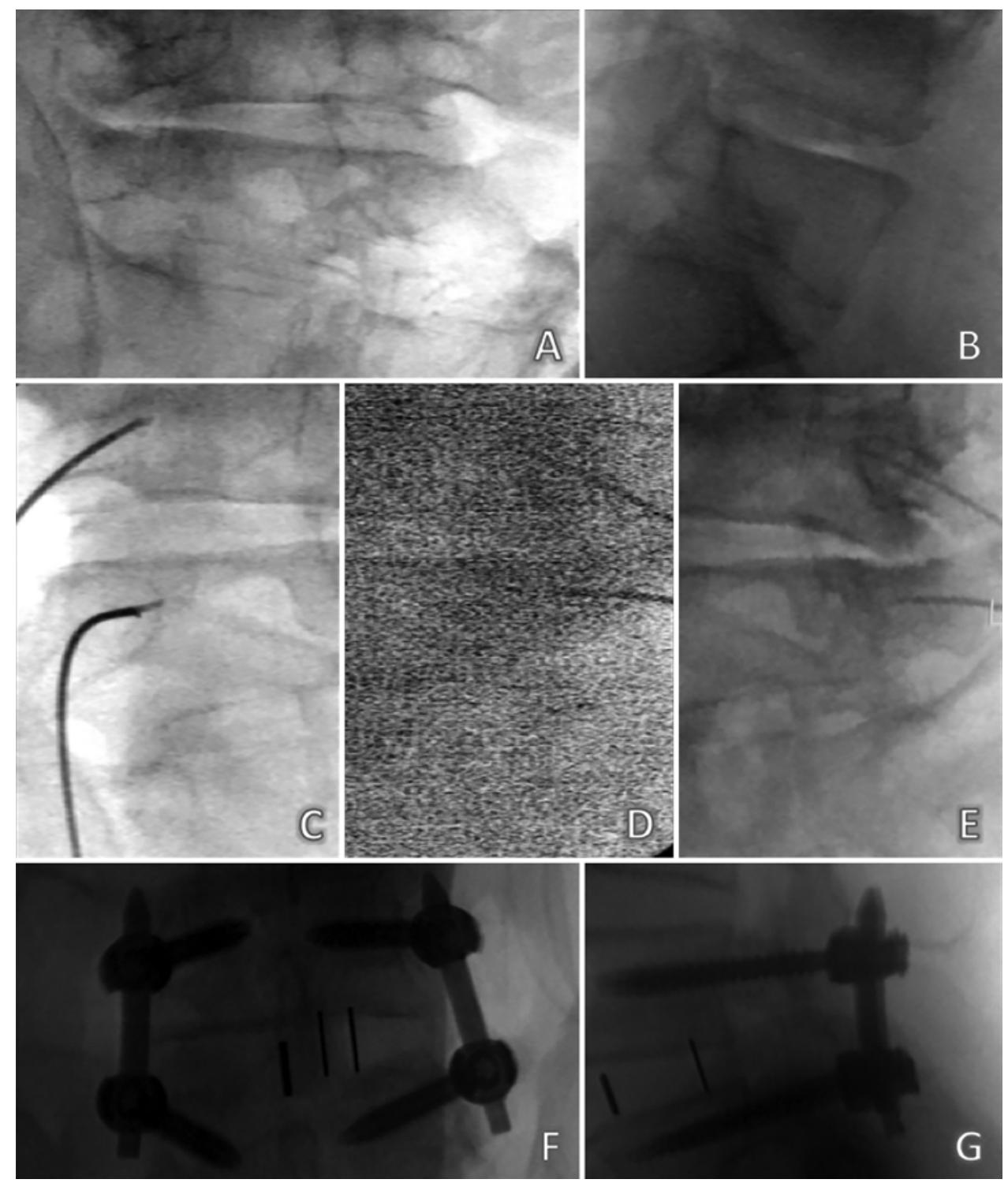

FIG. 2. Anteroposterior (A) and lateral (B) standard-dose fluoroscopy images taken preoperatively. K-wire placement and cannulation performed on the right side using standard-dose fluoroscopy $(\mathbf{C})$ versus the left side performed with ULRI and shown both before (D) and after (E) enhancement. Postoperative anteroposterior $(\mathbf{F})$ and lateral $(\mathbf{G})$ images are also shown.

tainer that started with an equal number of either outcome (Fig. 3).

Standard-dose fluoroscopy was performed using a OEC 9900 C-arm fluoroscopy unit (General Electric) with automatic brightness control and without technician modification, thereby approximating conventional surgeries. ULRI was performed in a similar manner, but without automatic brightness control. Instead, the $\mathrm{C}$-arm began each image set using a low-dose setting with 1 pulse/second imaging. Subsequently, the current was lowered during imaging until the surgeon felt the anatomical details were compromised by image quality.

The images from the ULRI group were then processed using LessRay, an FDA-approved tool for enhancing fluoroscopic images. Initially, the preoperative full-dose fluoroscopic image taken by the $\mathrm{C}$-arm was processed to form an improved image of the operative field. Starting with the anteroposterior position (Fig. 4), full-dose images are obtained to ensure that the patient was properly positioned with the spinous process midline between the pedicles of each level. Once the patient was confirmed to be positioned correctly, the fluoroscopy unit was placed in the lateral position. Lateral images were obtained initially at full-dose levels using LessRay. This system allows for a series of images to the "stitched" together to allow all of the levels being addressed to be viewed as 1 fused image. After the baseline images are fed into the LessRay system, the remainder of the surgical procedure can be performed using ULRI. The system even helps guide fluoroscopy to the exact position where the previous images were obtained, thereby ensuring that the subsequent image is obtained with the exact anatomy desired. This provides 


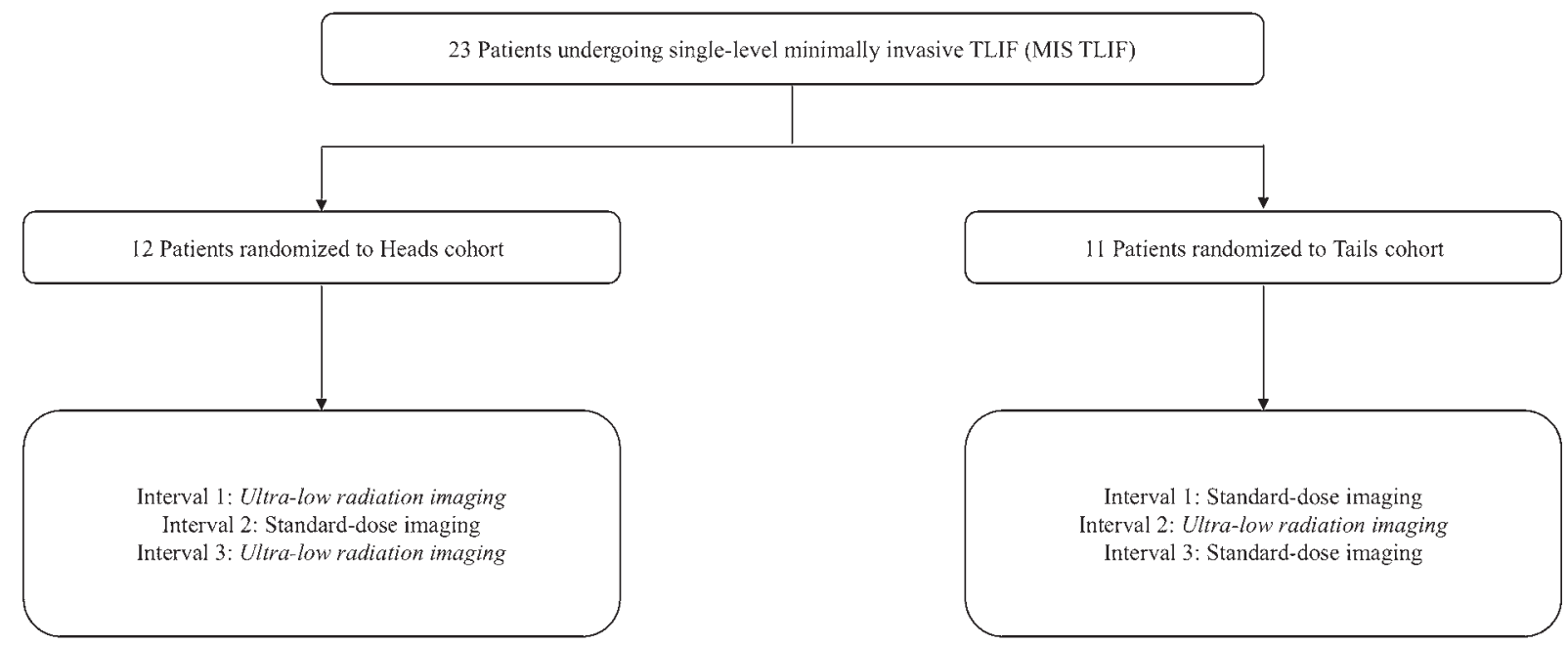

FIG. 3. Randomization of the patients into cohorts.

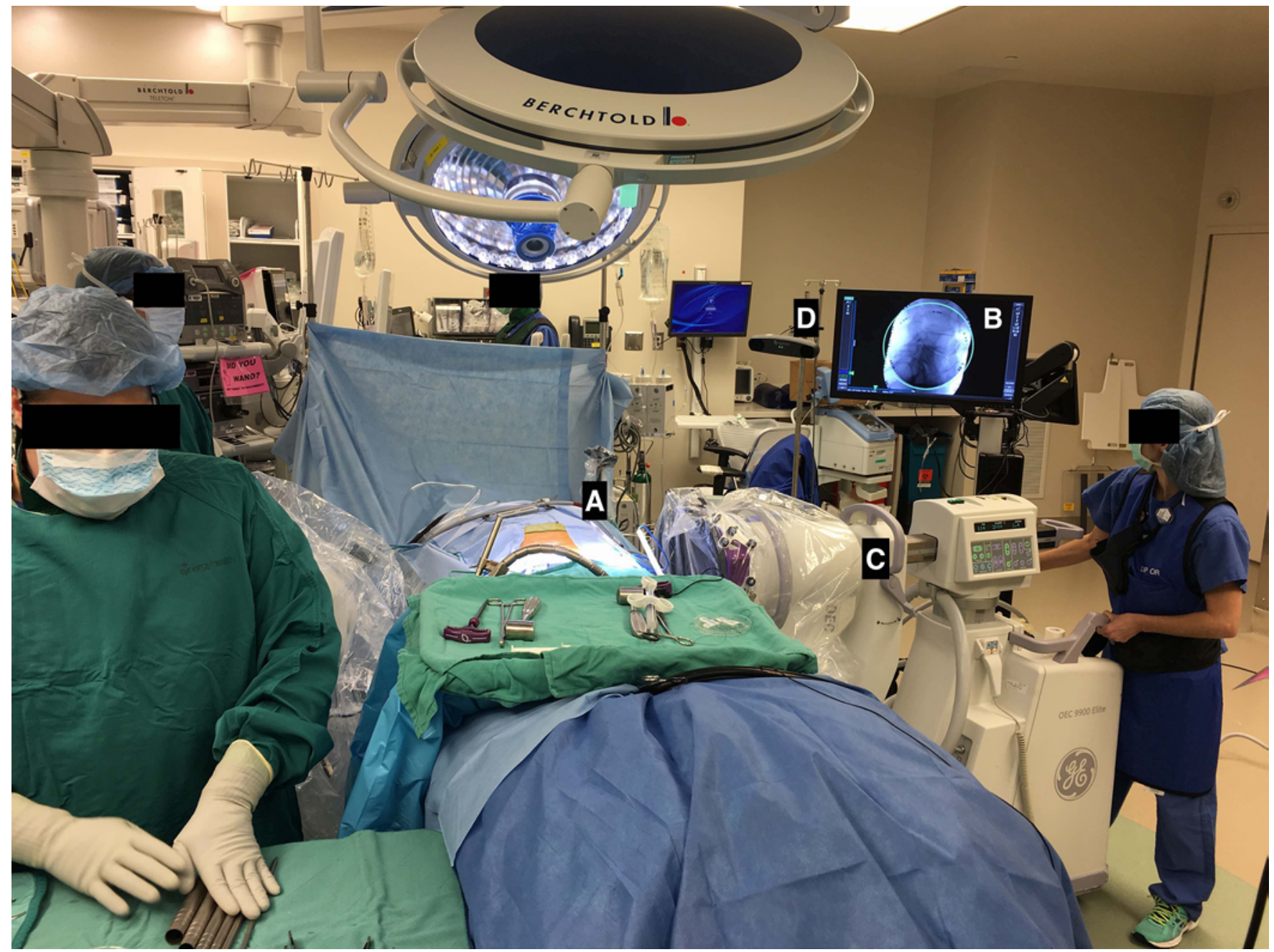

FIG. 4. Setup of the image enhancement system, including the infrared marker used by the LessRay infrared camera to locate the patient table (A), monitor showing the stitched baseline images taken from the lateral view (enhanced low-dose images are later displayed on this monitor) (B), C-arm in the lateral position taking full-dose preoperative images (C), and infrared camera for the detection of the location of the patient table, C-arm, and marked tools (D). Figure is available in color online only. 
TABLE 2. Demographic data of patients who underwent single-level MIS-TLIF with intraoperative imaging

\begin{tabular}{|c|c|c|c|c|}
\hline Variable & Total & Heads Group* & Tails Group $†$ & $p$ Value \\
\hline Age, mean $\pm S D$ & $59.09 \pm 16.25$ & $61.33 \pm 11.74$ & $56.64 \pm 20.40$ & 0.51 \\
\hline Male patients, n (\%) & $17(74)$ & $8(67)$ & $9(82)$ & 0.50 \\
\hline BMI, mean \pm SD & $29.52 \pm 4.19$ & $29.15 \pm 4.70$ & $29.92 \pm 3.74$ & 0.66 \\
\hline Spinal levels & 23 & 12 & 11 & \\
\hline L3-4 & 2 & 0 & 2 & \\
\hline L4-5 & 17 & 12 & 5 & \\
\hline L5-S1 & 4 & 0 & 4 & \\
\hline Rt-side TLIF, $n$ & 12 & 8 & 4 & 0.15 \\
\hline
\end{tabular}

nearly equivalent image quality to the original high-dose image using only low-dose exposures to obtain the subsequent images. This dramatically reduces the overall radiation used during the remainder of the surgical procedure. This not only improves the surgeon's ability to visualize the necessary anatomy and allows for the image to be obtained in a reproducible manner but also provides a live display of the instruments in the surgical field, such as Kwires and pedicle screws, with submillimetric spatial accuracy.

Intraoperative images for analysis were gathered after surgery from the General Electric OEC 9900 C-arm fluoroscopic unit and from LessRay. In addition, postoperative CT was performed using the O-arm (Medtronic Sofamor Danek) to better characterize pedicle screw accuracy and ensure high sensitivity for ascertaining pedicle breach.

\section{Analysis}

Images were then analyzed by 3 independent evaluators, all experienced spine surgeons, who were blinded as to which imaging modality was used to place each screw. The primary outcome measured was pedicle breach, which was graded by the accepted definitions of breach grades: Grade 0 (within $2.0 \mathrm{~mm})$, Grade $1(2.0-4.0 \mathrm{~mm})$, and Grade $2(4.0-6.0 \mathrm{~mm}){ }^{2,4}$ For each screw, the highest grade from among all 3 evaluators was taken as the breach grade to avoid underestimating the breach rates. Additionally, for any screw that breached $(>0.0 \mathrm{~mm})$, evaluators noted whether the screw breached medially or laterally. The effective breach rate was calculated as the percentage of screws placed that were evaluated as breached $>2.0$ $\mathrm{mm}$ medially or were postoperatively symptomatic. This definition is based on findings in the literature that suggest lateral breaches and breaches less than $2.0 \mathrm{~mm}$ are rarely symptomatic.,14 Screw depth and the angle of insertion were also analyzed. Appropriate depth was defined as insertion to $50 \%-75 \%$ of the vertebral body's anteroposterior dimension. The appropriate angle was defined as the screw angle of insertion being within $10^{\circ}$ of the pedicle angle.

Continuous variables were aggregated as the mean and standard deviation and analyzed using the Student t-test. Categorical variables were aggregated as proportions and analyzed by the Fisher exact test. Fleiss's kappa coefficient ( $\kappa)$ was calculated to test interobserver variability in pedicle breach grade. Significance was set at $p<0.05$ for all tests. All statistical analyses were done using JMP 13 (SAS Inc.) and Microsoft Excel 2016 (Microsoft Inc.).

\section{Results}

There were 23 patients (mean \pm SD age $59.09 \pm 16.25$ years; $74 \%$ of patients were men; mean \pm SD BMI 29.52 $\pm 4.19 \mathrm{~kg} / \mathrm{m}^{2}$ ) who underwent single-level MIS-TLIF between April 2015 and February 2016 and met the inclusion criteria for this study. The heads and tails cohorts had no significant differences in the baseline demographics and operated spinal levels (Table 2). Additionally, there was no significant difference between conventional fluoroscopy and ULRI in time spent on K-wire placement and cannulation (mean 10.57 vs 10.87 minutes; $\mathrm{p}=0.845$ ) and pedicle screw placement (mean 11.45 vs 23.82 minutes; $p$ $=0.073$ ). The total number of images taken was predicated on what was required for placement of instrumentation and was independent of the method used. The total fluoroscopy time was not recorded on the machine, as this was published prior when using ULRI and has been shown to be disassociated with radiation dose in low-dose protocols. ${ }^{17}$ Ultimately, the total decrease in radiation (Table 1) and surgical time (statistically identical) were the relevant factors to be analyzed.

In comparing screw placement by conventional fluoroscopy versus ULRI with image enhancement, no differences were seen in accuracy. If pedicle screw placement is analyzed by the imaging modality used for K-wire placement and cannulation (Table 3), no significant variations are seen in the breach grades between ULRI with image enhancement and conventional fluoroscopy. Similarly, no variations between these methods are seen if the pedicle screws are analyzed by the imaging modality used during screw placement itself (Table 4). The majority of screws $(95.6 \%)$ were evaluated as Grade $0(<2.0 \mathrm{~mm})$ and without medial deviation. While 2 screws $(4.3 \%)$ were evaluated as Grade $1(2.0-4.0 \mathrm{~mm})$, both breaches deviated laterally. Additionally, there were 2 screws (4.3\%) that deviated medially, but both were evaluated as Grade $0(<2.0 \mathrm{~mm})$. All 
TABLE 3. Pedicle screw placement accuracy based on the imaging modality used during K-wire placement and cannulation

\begin{tabular}{lccc}
\hline \multicolumn{1}{c}{ Variable } & ULRI & $\begin{array}{c}\text { Conventional } \\
\text { Fluoroscopy }\end{array}$ & $\begin{array}{c}\mathrm{p} \\
\text { Value }\end{array}$ \\
\hline No. of screws & 46 & 46 & \\
\hline Grade & & & \\
\hline Grade 0 & $44(95.6)$ & $46(100)$ & 0.153 \\
\hline Grade 1 & $2(4.3)$ & $0(0)$ & \\
\hline Grade 2 & $0(0)$ & $0(0)$ & \\
\hline Deviation & & & \\
\hline Medial & $2(4.3)$ & $0(0)$ & 0.157 \\
\hline Lateral & $5(10.9)$ & $0(0)$ & 0.220 \\
\hline Effective breach rate, $\%$ & 0 & 0 & 1 \\
\hline Poor angle & $0(0)$ & $0(0)$ & 1 \\
\hline Poor depth & $2(4.3)$ & $2(4.3)$ & 1 \\
\hline
\end{tabular}

Values are shown as the number of screws (\%) unless otherwise indicated.

4 of these screws had K-wire placement and cannulation performed under ULRI and screw placement performed under conventional fluoroscopy.

Furthermore, none of the 23 patients required revision surgery or experienced postoperative complications such as nerve damage, back pain, or CSF leak. Therefore, no screws met the criteria for evaluation as breached because there were no breaches $>2.0 \mathrm{~mm}$ medially and no clinical complications. As such, the effective breach rate for both imaging modalities was $0 \%$.

There was variability between the ratings for breaches, with the $\kappa$ coefficient indicating only fair agreement $(\kappa$ $=0.226$ ). However, the percentage of agreement was far higher, with all 3 evaluators agreeing $92.4 \%$ of the time. The low $\kappa$ coefficient, despite the high percentage of agreement, indicates that evaluators strongly disagreed on a small minority of screws. In analyzing the images that led to disagreement (representative image shown in Fig. 5), it was found that the postoperative CT image in each case was low quality, which could prevent consensus regarding the accuracy of screw placement. Given this variability, the worst grade assigned to any screw by any evaluator was used to avoid overestimating accurate placement.

In evaluating screw depth, 1 observer noted 1 operation wherein all 4 screws did not achieve adequate depth (50\%$75 \%$ of the screw's length). For K-wire placement and cannulation, 2 of these screws were placed using URLI and 2 were placed using conventional fluoroscopy $(\mathrm{p}=1.00)$. For pedicle placement, all 4 of these screws were placed using ULRI $(\mathrm{p}=0.034)$. However, the $\kappa$ coefficient for interobserver variability indicated no agreement $(\kappa=-0.01)$ because the other 2 evaluators felt that all 4 screws had an appropriate depth. Additionally, no screws were noted to have an inappropriate angle $(\mathrm{p}=1.00)$.

\section{Discussion}

This internally randomized controlled trial provides novel, Level 1 evidence that ULRI with image enhancement is as safe and accurate as conventional, standard-
TABLE 4. Pedicle screw placement accuracy based on the imaging modality used during pedicle screw placement

\begin{tabular}{lccc}
\hline \multicolumn{1}{c}{ Variable } & ULRI & $\begin{array}{c}\text { Conventional } \\
\text { Fluoroscopy }\end{array}$ & $\begin{array}{c}p \\
\text { Value }\end{array}$ \\
\hline No. of screws & 44 & 48 & \\
\hline Grade & & \\
\hline Grade 0 & $44(100)$ & $46(95.8)$ & 0.171 \\
\hline Grade 1 & $0(0)$ & $2(4.2)$ & \\
\hline Grade 2 & $0(0)$ & $0(0)$ & \\
\hline Deviation & & \\
\hline Medial & $0(0)$ & $2(4.2)$ & 0.172 \\
\hline Lateral & $3(6.8)$ & $2(4.2)$ & 0.585 \\
\hline Effective breach rate, \% & 0 & 0 & 1 \\
\hline Poor angle & $0(0)$ & $0(0)$ & 1 \\
\hline Poor depth & $4(9.1)$ & $0(0)$ & $0.034^{*}$ \\
\hline $\begin{array}{l}\text { Values are shown as the number of screws (\%) unless otherwise indicated. } \\
\text { * Statistically significant at } p<0.05 .\end{array}$
\end{tabular}

dose fluoroscopy for pedicle screw placement. The effective breach rate for both imaging modalities was $0 \%$.

The costs of poor pedicle screw placement and subsequent breaches are well established. In a prospective study of 200 patients undergoing thoracolumbar pedicle screw instrumentation, Watkins et al. estimated that revision surgery for a pedicle breach costs about $\$ 23,700$ at their institution. ${ }^{22}$ Furthermore, while percutaneous screw placement during minimally invasive procedures has well-established benefits for patients, ${ }^{11,13,15}$ the procedure remains technically demanding due to the lack of direct visualization and poor tactile feedback during placement. ${ }^{20}$

Intraoperative imaging provides a powerful tool for improving the accuracy of pedicle screw placement during surgical fusion. In a meta-analysis of 130 studies by Kosmopoulos and Schizas, the rates of successful screw insertion into the pedicle were significantly higher when performed utilizing intraoperative imaging and navigation systems versus freehand insertion. ${ }^{8}$ As minimally invasive approaches grow more prevalent, there is an increased reliance on imaging support systems to adequately visualize the surgical window and reduce the rate of pedicle breach. ${ }^{5,6}$ Additionally, variations occur with different imaging modalities, and a meta-analysis by Tian et al. of 43 studies found that both 3D fluoroscopy and CT have shown improved breach rates compared with conventional 2D fluoroscopy. ${ }^{16}$

Whichever imaging modality is used, there remains a significant increase in radiation exposure for both the patient and operating room personnel..$^{3,12,19,21}$ In a prospective study of 158 hospital workers, Mastrangelo et al. found that orthopedic surgeons had a significantly higher risk of developing cancer due to radiation exposure..$^{12}$ In a prospective study of radiation exposure to an index spine surgeon utilizing fluoroscopy, Ul Haque et al. showed that exposure would exceed the National Council on Radiation Protection's lifetime limit for radiation within the first 10 years of the surgeon's career. ${ }^{18}$ Furthermore, a meta-analysis of 8 cohort studies by Kim et al. found that patient exposure is greater with MIS-TLIF versus conventional, open TLIF 

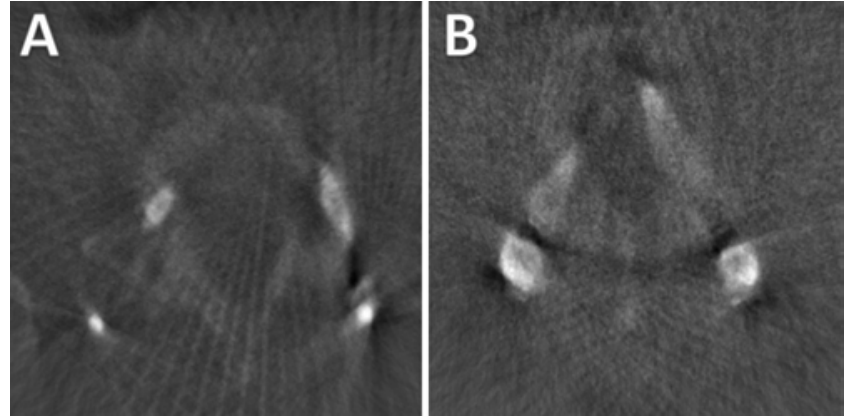

FIG. 5. A: Poor-quality postoperative scan showing the right pedicle screw judged to be deviated medially $<2.0 \mathrm{~mm}$ by 1 evaluator and as not deviated by 2 evaluators. B: Right pedicle screw judged to be deviated laterally $2.0-4.0 \mathrm{~mm}$ by 1 evaluator, $<2.0 \mathrm{~mm}$ by another evaluator, and as not deviated by the last evaluator. Because the bony anatomy is poorly visualized on these scans, pedicle screw placement is challenging to evaluate. All images that led to evaluator disagreement on screw placement accuracy were similarly obfuscated.

(range $0.64-0.66 \mathrm{mSv}$ in open TLIF vs $1.54-1.63 \mathrm{mSv}$ in MIS-TLIF). ${ }^{6}$ This increase in radiation exposure is all the more important given that the patient's understanding in this area is often poor, with many patients underestimating their intraoperative exposure. ${ }^{1}$

In a previous study, Wang et al. found that image enhancement with ULRI can significantly decrease radiation exposure to operating room staff and patients. ${ }^{21}$ This study presents evidence from an internally randomized controlled trial indicating that ULRI with image enhancement provides adequate visualization for accurate pedicle screw placement. No significant differences in the breach rate or postoperative complications were found in the pedicle screws placed under ULRI with image enhancement compared with conventional fluoroscopy. Significant variations in pedicle screw evaluation were seen between observers, with the $\kappa$ coefficient indicating only fair agreement. This may partly be due to poor imaging, as postoperative CT scans from these cases were decidedly compromised in quality and had high levels of artifact (Fig. 5). Furthermore, a retrospective study of 286 pedicle screws by Lavelle et al. found that even experienced spine surgeons evaluating high-quality postoperative CT scans for breach had significantly high intra- and interobserver variability. ${ }^{10}$

In this study, 2 screws were noted to be deviated medially, although these breaches were Grade $0(<2.0 \mathrm{~mm})$. While medial breaches are important because intrusion into the central canal can occur, previous studies have suggested that smaller breaches are largely inconsequential. In a retrospective study of 1218 pedicle screws, Koktekir et al. suggested that there was a $2.0-\mathrm{mm}$ safety zone medially wherein breaches caused no symptoms. ${ }^{7}$ Additionally, in a study of imaging by Reynolds et al., the distance from the pedicle screw to the dura was about $2 \mathrm{~mm}$ from T-7 to L-4, so perforations in this area were asymptomatic. ${ }^{14}$ Therefore, the 2 medial breaches observed in this study were not considered critical and unlikely to perforate the central canal.

This study also identified 5 lateral breaches, with 2 placements assessed as Grade 1 (2.0-4.0 mm). However, lateral breaches are often reported as asymptomatic. In a prospective study of 40 consecutive patients who were surgically treated for thoracolumbar spinal deformity, Gertzbein et al. found that lateral breaches occurred $3.7 \%$ of the time and were not symptomatic. ${ }^{4}$ Additionally, a prospective study of 152 screws in 30 low-back spinal surgeries by Laine et al. found that any breach that perforated less than $4.0 \mathrm{~mm}$ was clinically inconsequential and did not cause any neurological deficits. ${ }^{9}$ Because all of the lateral breaches observed in this study had less than $4.0 \mathrm{~mm}$ of perforation, were asymptomatic, and did not require revision surgery, none were considered critical. Therefore, all breaches noted in this study were deemed noncritical, yielding an overall effective breach rate of $0 \%$ independent of the imaging modality used.

While this is the first clinical study to show that ULRI with image enhancement is an effective imaging technique for accurate screw placement, there are limitations that impact the interpretation of results. Although this study showed no variations in screw placement across 23 patients and 92 screws, analysis of a larger sample may reveal more subtle trends in screw placement accuracy between imaging modalities. Additionally, while utilizing a single surgeon helps standardize the operative course, the surgeon was not blinded to the imaging modality being used, which could impact surgical outcomes. Because this study also only reflects a single surgeon's experience with 23 patients, future studies should assess a larger cohort. Furthermore, while patients were monitored for postoperative complications and no revision surgeries were required, no patientreported outcomes were collected to assess differences in postoperative pain, disability, and satisfaction. Finally, there was disagreement among evaluators that coincided with the cases that had poor-quality postoperative CT scans. Despite these limitations, this study indicates that ULRI with image enhancement is an effective tool for accurate pedicle screw placement and provides a proven decrease in radiation exposure to both patients and operating room staff.

\section{Conclusions}

ULRI with image enhancement allows for accurate pedicle screw placement during MIS-TLIF. Compared with conventional fluoroscopy, no significant differences were seen in the effective pedicle screw breach rate. As previously reported, this image enhancement protocol drastically reduces radiation exposure to patients and operating room staff when compared with standard-dose fluoroscopy. Given the evidence in both randomized controlled trials, this study concludes that ULRI with image enhancement provides visualization for safe and efficacious pedicle screw placement while significantly minimizing radiation exposure.

\section{References}

1. Bohl DD, Hijji FY, Massel DH, Mayo BC, Long WW, Modi $\mathrm{KD}$, et al: Patient knowledge regarding radiation exposure from spinal imaging. Spine J 17:305-312, 2017

2. Gautschi OP, Schatlo B, Schaller K, Tessitore E: Clinically relevant complications related to pedicle screw placement in thoracolumbar surgery and their management: a literature 
review of 35,630 pedicle screws. Neurosurg Focus 31(4):E8, 2011

3. Gebhard FT, Kraus MD, Schneider E, Liener UC, Kinzl L, Arand M: Does computer-assisted spine surgery reduce intraoperative radiation doses? Spine (Phila Pa 1976) 31:20242028, 2006

4. Gertzbein SD, Robbins SE: Accuracy of pedicular screw placement in vivo. Spine (Phila Pa 1976) 15:11-14, 1990

5. Khanna R, McDevitt JL, Abecassis ZA, Smith ZA, Koski TR, Fessler RG, et al: An outcome and cost analysis comparing single-level minimally invasive transforaminal lumbar interbody fusion using intraoperative fluoroscopy versus computed tomography-guided navigation. World Neurosurg 94:255-260, 2016

6. Kim $\mathrm{CH}$, Lee $\mathrm{CH}$, Kim KP: How high are radiation-related risks in minimally invasive transforaminal lumbar interbody fusion compared with traditional open surgery?: a metaanalysis and dose estimates of ionizing radiation. Clin Spine Surg 29:52-59, 2016

7. Koktekir E, Ceylan D, Tatarli N, Karabagli H, Recber F, Akdemir G: Accuracy of fluoroscopically-assisted pedicle screw placement: analysis of 1,218 screws in 198 patients. Spine $\mathbf{J}$ 14:1702-1708, 2014

8. Kosmopoulos V, Schizas C: Pedicle screw placement accuracy: a meta-analysis. Spine (Phila Pa 1976) 32:E111-E120, 2007

9. Laine T, Mäkitalo K, Schlenzka D, Tallroth K, Poussa M, Alho A: Accuracy of pedicle screw insertion: a prospective CT study in 30 low back patients. Eur Spine J 6:402-405, 1997

10. Lavelle WF, Ranade A, Samdani AF, Gaughan JP, D'Andrea LP, Betz RR: Inter- and intra-observer reliability of measurement of pedicle screw breach assessed by postoperative CT scans. Int J Spine Surg 8: 2014

11. Lowery GL, Kulkarni SS: Posterior percutaneous spine instrumentation. Eur Spine J 9 (Suppl 1):S126-S130, 2000

12. Mastrangelo G, Fedeli U, Fadda E, Giovanazzi A, Scoizzato L, Saia B: Increased cancer risk among surgeons in an orthopaedic hospital. Occup Med (Lond) 55:498-500, 2005

13. Park Y, Ha JW: Comparison of one-level posterior lumbar interbody fusion performed with a minimally invasive approach or a traditional open approach. Spine (Phila Pa 1976) 32:537-543, 2007

14. Reynolds AF Jr, Roberts PA, Pollay M, Stratemeier PH: Quantitative anatomy of the thoracolumbar epidural space. Neurosurgery 17:905-907, 1985

15. Ringel F, Stoffel M, Stüer C, Meyer B: Minimally invasive transmuscular pedicle screw fixation of the thoracic and lumbar spine. Neurosurgery 59 (4 Suppl 2):ONS361-ONS367, 2006

16. Tian NF, Huang QS, Zhou P, Zhou Y, Wu RK, Lou Y, et al: Pedicle screw insertion accuracy with different assisted methods: a systematic review and meta-analysis of comparative studies. Eur Spine J 20:846-859, 2011
17. Tumialán LM, Clark JC, Snyder LA, Jasmer G, Marciano FF: Prospective evaluation of a low-dose radiation fluoroscopy protocol for minimally invasive transforaminal lumbar interbody fusion. Oper Neurosurg 11:537-544, 2015

18. Ul Haque M, Shufflebarger HL, O'Brien M, Macagno A: Radiation exposure during pedicle screw placement in adolescent idiopathic scoliosis: is fluoroscopy safe? Spine (Phila Pa 1976) 31:2516-2520, 2006

19. Villard J, Ryang YM, Demetriades AK, Reinke A, Behr M, Preuss A, et al: Radiation exposure to the surgeon and the patient during posterior lumbar spinal instrumentation: a prospective randomized comparison of navigated versus non-navigated freehand techniques. Spine (Phila Pa 1976) 39:1004-1009, 2014

20. Voyadzis JM: The learning curve in minimally invasive spine surgery. Semin Spine Surg 23:9-13, 2011

21. Wang TY, Farber SH, Perkins SS, Back AG, Byrd SA, Chi $\mathrm{D}$, et al: An internally randomized control trial of radiation exposure using ultra-low radiation imaging (ULRI) versus traditional C-arm fluoroscopy for patients undergoing singlelevel minimally invasive transforaminal lumbar interbody fusion (TLIF). Spine (Phila Pa 1976) [epub ahead of print], 2016

22. Watkins RG, Gupta A, Watkins RG: Cost-effectiveness of image-guided spine surgery. Open Orthop J 4:228-233, 2010

\section{Disclosures}

Dr. Karikari: consultant for NuVasive.

\section{Author Contributions}

Conception and design: Karikari, Nayar. Acquisition of data: Karikari, Nayar, Back, Vincent. Analysis and interpretation of data: Karikari, Nayar, Blizzard, Cook. Drafting the article: Nayar, Blizzard, Wang, Back, Vincent. Critically revising the article: Karikari, Nayar, Wang, Back, Vincent. Reviewed submitted version of manuscript: all authors. Approved the final version of the manuscript on behalf of all authors: Karikari. Statistical analysis: Nayar, Wang, Vincent.

\section{Supplemental Information}

\section{Previous Presentations}

Portions of this work were presented in abstract form at the 2017 American Association of Neurological Surgeons Annual Scientific Meeting in Los Angeles, California, on April 23, 2017.

\section{Correspondence}

Isaac O. Karikari, Duke University Medical Center, Box 3807, Durham, NC 27710. email: isaac.karikari@duke.edu. 